\title{
EFECTO EN LA RESISTENCIA DE LAS ESCORIAS DE FUNDICIÓN DE COBRE COMO AGREGADO FINO EN EL COMPORTAMIENTO RESISTENTE DEL HORMIGÓN
}

\section{EFFECT OF SMELTING COPPER SLAG AS FINE AGGREGATE ON THE RESISTANT BEHAVIOR OF CONCRETE}

\author{
Patricio Cendoya ${ }^{1}$ \\ Recibido 9 de julio de 2007, aceptado 5 de marzo de 2009 \\ Received: July 9, 2007 Accepted: March 5, 2009
}

\begin{abstract}
RESUMEN
Las escorias de fundición de cobre son residuos industriales provenientes de la fundición del cobre, las cuales procesadas en forma de granallas y sometidas a un proceso de molienda adquieren características similares a las de un árido fino. La presente investigación estudia la influencia que tiene su incorporación en el comportamiento mecánico a flexotracción y compresión en hormigones que emplean como árido fino una combinación de arenas del río Bío-Bío con proporciones de $25 \%, 40 \%$ y $50 \%$ en volumen de escorias de fundición de cobre. El árido fino resultante se utiliza en la confección de hormigones dosificados para relaciones de agua cemento de 0,45 y 0,52 asociadas a resistencias especificadas a la flexotracción de 3,6 y 4,3 MPa. Se mide la trabajabilidad en el hormigón fresco, la densidad, la carga de rotura por flexotracción y la carga de rotura por compresión en el hormigón endurecido comparando los resultados con un hormigón de referencia que no contiene escorias. Los resultados señalan que la docilidad de la mezcla se incrementa debido a la textura lisa de las escorias, se produce un aumento de la densidad del hormigón endurecido y las resistencias tanto a flexotracción como compresión se incrementan en función del contenido de escorias de fundición de cobre utilizado en la mezcla.
\end{abstract}

Palabras clave: Hormigones, escorias de fundición de cobre, resistencia a la flexotracción y compresión.

\begin{abstract}
Copper slag is a by product of the copper smelting industry. We took granulated copper slag and milled it until it acquired characteristics similar to those of a fine aggregate, which was then incorporated into concrete, in combination with Bio-Bío river sand in proportions of 25\%, 40\% and 50\% by volume of copper slag. We then examined the impact of the different combinations on the mechanical behaviour to flexotraction and compression in the resulting concrete. Our specification was to produce concrete for water-cement ratios of 0.45 and 0.52 associated to breakage resistances to flexotraction of 3.6 and $4.3 \mathrm{MPa}$, respectively. We measured workability of the fresh concrete, as well as density, and breaking load by flexotraction and compression in the hardened concrete, comparing results with a concrete control that did not contain smelting copper slag. Results indicate that the mixture's docility increases due to the smoother texture of the slag. Concrete density and hardened resistance to compression and flexotraction also increase, depending on the content of slag copper smelter used in the mix.
\end{abstract}

Keywords: Concrete, copper melting slags, flexotraction and compression resistance.

\section{INTRODUCCIÓN}

En las últimas décadas la economía chilena ha estado dominada por la producción de cobre. Esto se hace notar con la existencia en Chile de siete fundiciones de cobre Chuquicamata (Calama), Alto Norte (Antofagasta), Potrerillos (El Salvador), Paipote (Copiapó), Hernán
Videla Lira (Copiapó), Ventanas (Los Andes) y Caletones (Rancagua). Cada una de estas fundiciones genera grandes volúmenes de residuos, desechos y subproductos, entre los que encontramos polvos de fundición, ácido sulfúrico y escorias de fundición de cobre (EFC). Actualmente Chile es uno de los mayores productores de cobre de fundición en el mundo, a través de sus siete fundiciones

\footnotetext{
1 Departamento de Ingeniería Civil, Universidad de Concepción, Casilla 160-C, Correo 3. Concepción, Chile. E-mail: pcendoya@udec.cl
} 
que produjeron 1,6 millones toneladas de cobre (13\% del total mundial) en el 2005, mientras cerca de 3,5 millones toneladas de escorias son producidas anualmente [1]. Por cada tonelada de cobre obtenido se generan 2,2 toneladas de escorias pirometalúrgicas. Se estima que cada año se producen 4,5 millones de toneladas de estos residuos y que el volumen histórico acumulado bordea los 50 millones [1, 2].

La utilización de EFC en la industria de la Construcción no es nueva. A nivel nacional se han utilizado como rellenos de caminos (estabilización de asfaltos), en la fabricación de lanas minerales (para aislamiento), en la fabricación de ladrillos refractarios y como material abrasivo para limpieza de superficies de acero [2].

A nivel internacional (Canadá, Estados Unidos) se ha utilizado con buenos resultados como base granular en la construcción de caminos, líneas férreas y terraplenes [3]. En Brasil, se ha estudiado la influencia de la utilización de EFC como adición en el cemento y como agregado fino [4]. En Japón, se han realizado investigaciones para el uso de diversos tipos de desechos industriales, incluyendo las EFC como agregado en el hormigón [5].

Diversos investigadores $[4,6]$ estudian el comportamiento y el efecto de incluir escorias de fundición de cobre como agregado fino en el hormigón. Al-Jabri [6] confecciona mezclas de hormigón con distintas proporciones de escoria de cobre, como reemplazo parcial y total del agregado fino, utilizando como porcentaje de reemplazo en peso desde un 10 hasta un $100 \%$ de la arena utilizada. Para cada tipo de mezcla se desarrollan ensayos para determinar la resistencia a la compresión, tracción y flexión. Los resultados muestran un aumento de la densidad y de la trabajabilidad con respecto a hormigones normales y en general un aumento de la resistencia a medida que aumenta la cantidad de escoria de cobre; sin embargo, la resistencia crece hasta cierto contenido de ella, ya que con cantidades más elevadas, comienza a disminuir. Las mezclas con elevados porcentajes de escorias de cobre presentan muestras de exudación y segregación debido al significativo aumento de la trabajabilidad.

Moura [4] utiliza porcentajes de reemplazo del árido fino del 30\%, 40\% y 50\% para distintas razones de agua cemento realizando ensayos de compresión y flexotracción. Para el hormigón en estado fresco encuentra un aumento de la trabajabilidad y en el peso específico. En relación a las características del hormigón en estado endurecido, el peso específico se ve incrementado en el orden de un $10 \%$ con respecto al hormigón de referencia cuando utiliza un $50 \%$ de escoria de cobre como reemplazo del agregado fino. Con respecto a la resistencia a la flexotracción esta se incrementa en un $10 \%$ con respecto al hormigón de referencia cuando se utiliza un porcentaje de reemplazo de $40 \%$ de escoria.

Zapata [7], realiza un estudio experimental sobre el efecto que tiene sobre la resistencia a la compresión el hecho de utilizar EFC como árido fino. Considera distintas proporciones en volumen de $\operatorname{EFC}(25 \%$ y $50 \%)$ en reemplazo de igual porcentaje de árido fino (arena) para relaciones de agua cemento de 0,4 y 0,5. Los resultados indican que los hormigones que utilizan una proporción de EFC como árido fino presentan características similares de trabajabilidad y mejoran su resistencia a la compresión simple en relación a los hormigones que únicamente utilizan arena como agregado fino [8].

\section{INVESTIGACIÓN PROPUESTA}

El propósito de la presente investigación se centra en el estudio experimental de la resistencia a la flexotracción de hormigones fabricados con un árido fino obtenido a partir de la combinación de arena Bío-Bío con granalla de EFC en distintas proporciones en volumen $(25 \%$, $40 \%$ y $50 \%)$ para dos relaciones de agua cemento $(0,45$ y 0,52) [9]. A través de este estudio se busca validar las experiencias previas desarrolladas por Moura [4], AlJabri [6] en cuanto a la trabajabilidad en el hormigón fresco, la densidad y resistencia a la flexotracción en el hormigón endurecido cuando se utilizan como materia prima granallas de EFC provenientes de la fundición Caletones (El Teniente, Codelco, Chile).

\section{MATERIALES Y MÉTODOS}

Materiales: la granalla de EFC posee una granulometría muy variada, con tamaños máximos del orden de $20 \mathrm{~mm}$ y mínimos de aproximadamente $1 \mathrm{~mm}$ de formas varias con tendencias a redondeadas y no alargadas, color negro, textura lisa y poco porosa, ver figura 1 . 


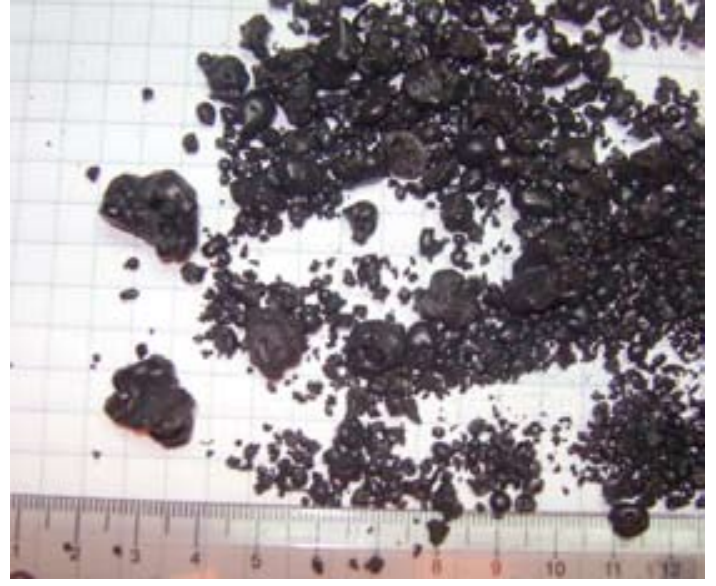

Figura 1. Granalla de EFC.

Sus características físicas más importantes se resumen en la tabla 1.

Tabla 1. Propiedades físicas de las granallas de EFC $[7,9]$.

\begin{tabular}{|l|c|}
\hline Densidad real seca $\left(\mathrm{kg} / \mathrm{m}^{3}\right)$ & 4.000 \\
\hline Densidad aparente suelta seca $\left(\mathrm{kg} / \mathrm{m}^{3}\right)$ & 2.236 \\
\hline Densidad aparente compacta seca $\left(\mathrm{kg} / \mathrm{m}^{3}\right)$ & 2.372 \\
\hline Absorción (\%) & 0,200 \\
\hline Módulo de finura & 3,16 \\
\hline Finos por lavado, bajo malla 200 (\%) & 2,08 \\
\hline
\end{tabular}

Un análisis granulométrico de la granalla de EFC disponible [7,9] arroja que esta no cumple con la banda granulométrica de la NCh 163 Of. 79 [10], siendo necesario realizar un proceso de molienda para ajustar su granulometría a los requerimientos de la norma y poder de esta forma utilizarla como árido fino en la confección de hormigones. Realizado el proceso de molienda, se obtiene un material que se ajusta a la banda granulométrica de la norma $\mathrm{NCh}$ 163 Of. 79 [10] tal como se aprecia en la figura 2.

El material resultante del proceso de molienda se combina en distintas proporciones en volumen (25\%, 40\% y 50\%) con arenas Bío-Bío de forma de obtener un árido fino combinado (AFC) que satisfaga las especificaciones de la NCh 163 Of. 79 [10], tal como se aprecia en la figura 3 .

Con relación a la gravilla y grava estas son provenientes del río Itata y el cemento utilizado es siderúrgico que cumple con la NCh 148 Of. 68 [11].

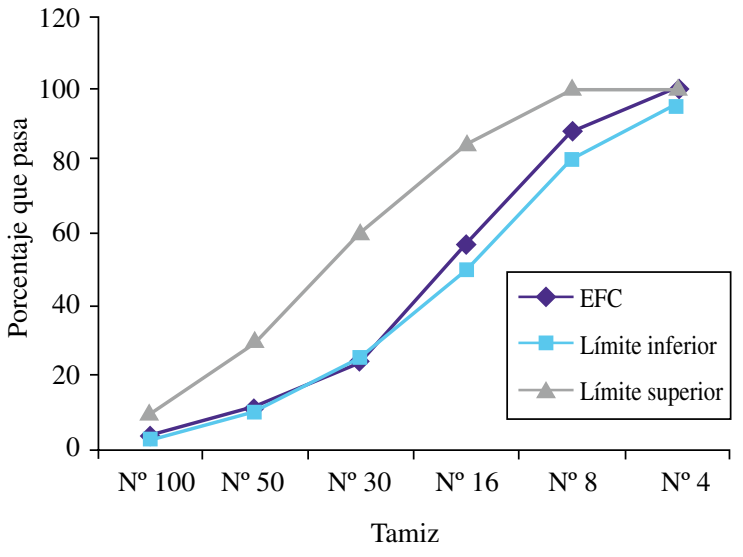

Figura 2. Curva granulométrica de granallas de EFC después del proceso de molienda. Límites de inferior y superior de NCh 163 Of. 79 [10].

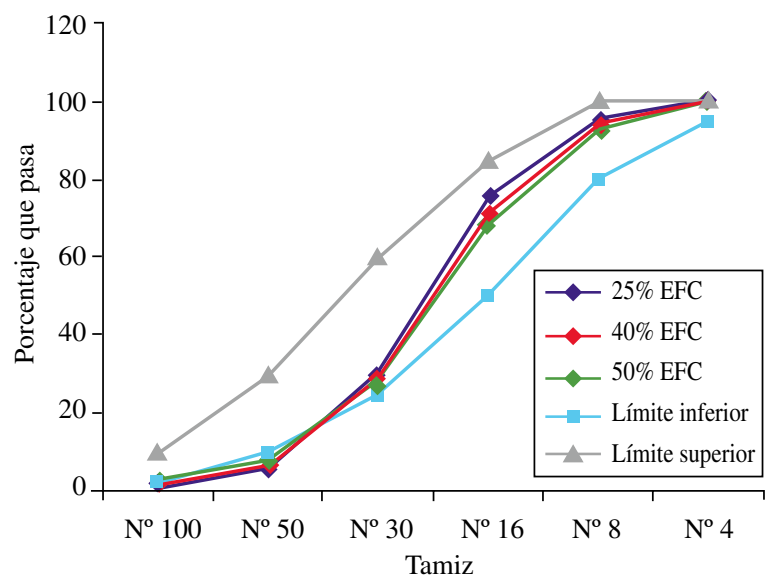

Figura 3. Curvas granulométricas del árido fino combinado.

Dosificación: utilizando el método propuesto por la norma NCh. 170 Of. 85 [12], se dosifica la mezcla considerando: una fracción defectuosa del $20 \%$. Una resistencia especificada a la flexotracción de 4,3 $\mathrm{MPa}$ a los 28 días para hormigones con relación agua cemento de 0,45 y una resistencia especificada a la flexotracción de 3,6 MPa a los 28 días para los hormigones con relación agua cemento de 0,52 . Se considera en ambas dosificaciones un asentamiento teórico del orden de 3 a $5 \mathrm{~cm}$.

En la tabla 2 se presenta un resumen de la dosificación empleada en la fabricación de coladas de 75 litros con relación de agua cemento de 0,45 y diferentes contenidos de EFC. 
Tabla 2. Dosificación para una relación A/C=0.45 [9].

\begin{tabular}{|c|c|c|c|c|}
\hline Materiales & $\mathbf{0 \%}$ EFC & $\mathbf{2 5 \%}$ EFC & $\mathbf{4 0 \%}$ EFC & 0\% EFC \\
\hline $\mathbf{A} / \mathbf{C}=\mathbf{0 , 4 5}$ & $\left(\boldsymbol{l} / \mathbf{m}^{\mathbf{3}}\right)$ & $\left(\boldsymbol{l} / \mathbf{m}^{\mathbf{3}}\right.$ & $\left(\boldsymbol{l} / \mathbf{m}^{\mathbf{3}}\right)$ & $\left(\boldsymbol{l} / \mathbf{m}^{\mathbf{3}}\right)$ \\
\hline Cemento & 118,5 & 118,5 & 118,5 & 118,5 \\
\hline Agua & 160,0 & 160,0 & 160,0 & 10,0 \\
\hline Aire & 10,0 & 10,0 & 10,0 & 110,3 \\
\hline Arena & 220,6 & 165,4 & 132,3 & 206,3 \\
\hline Gravilla & 206,3 & 206,3 & 206,3 & 284,6 \\
\hline Grava & 284,6 & 284,6 & 284,6 & 110,3 \\
\hline Escoria & 0,0 & 55,1 & 88,2 & $1.000,0$ \\
\hline Total & $1.000,0$ & $1.000,0$ & $1.000,0$ & \\
\hline
\end{tabular}

A/C=relación agua cemento.

Para cada porcentaje de EFC $(25 \%, 40 \%$ y $50 \%)$ y relación agua cemento $(0,45$ y 0,52$)$ se confeccionan dos muestras de hormigón: una de 60 litros y otra de 75 litros, obteniéndose en total 9 viguetas por cada combinación (2 para ser ensayadas a los 3 días, 2 para ser ensayadas a los 7 días, 4 para ser ensayadas a los 28 días y una extra por posibles eventualidades). El motivo de realizar dos muestras con las mismas características es entregar una mayor confiabilidad a los resultados experimentales. En el caso de los hormigones de referencia ( $0 \%$ de EFC), sólo se realiza una muestra de 75 litros por cada relación agua cemento $(0,45$ y 0,52$)$.

En resumen un total de 64 viguetas se confeccionan: 18 para un $25 \%$ de EFC (9 por cada relación agua cemento), 18 para un $40 \%$ de EFC ( 9 por cada relación agua cemento), 18 para $50 \%$ de EFC (9 por cada relación agua cemento) y 10 para el hormigón de referencia (5 por cada relación agua cemento), [9].

Identificación de probetas: con el fin de poder asociar cada vigueta con una determinada relación agua cemento $\mathrm{y}$ contenido de EFC, se define la siguiente nomenclatura para su identificación:

$$
H-A / C-\% E F C
$$

En donde:

La primera letra indica hormigón $(\mathrm{H})$, las siguientes tres letras señalan la relación agua cemento y las dos últimas corresponden al porcentaje de EFC.

Todas las probetas son etiquetadas para su posterior identificación, ver figura 4.

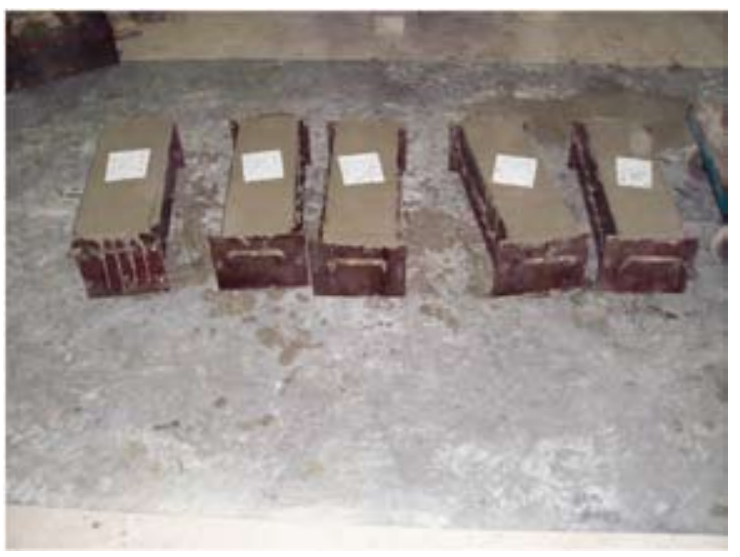

Figura 4. Identificación de probetas.

Curado: el curado de las probetas se realiza según NCh 1017 Of. 75 [13], en cámara húmeda en donde permanecen hasta la fecha del ensayo, siendo desmoldadas a las 48 horas de su confección.

Ensayo de flexotracción: siguiendo las indicaciones de NCh 1038 Of. 77 [14], se aplica la carga vertical en los tercios centrales de la viga a una velocidad constante de $1.4 \mathrm{kgf} / \mathrm{cm}^{2} / \mathrm{s}$, ver figura 5 .

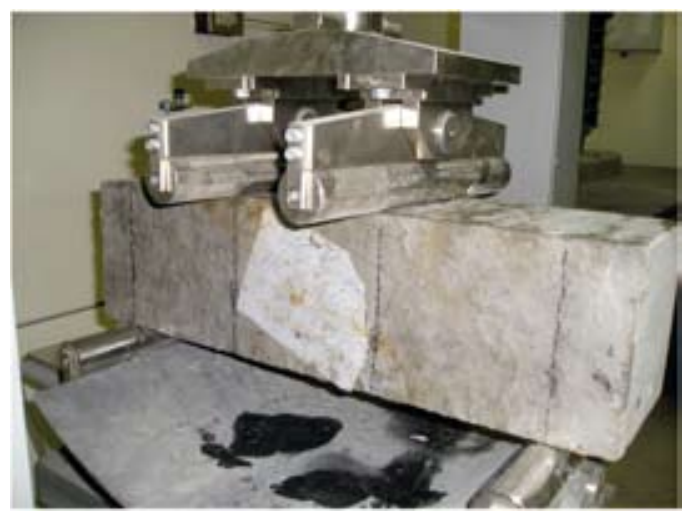

Figura 5. Esquema del ensayo a flexotracción. 


\section{RESULTADOS}

Del total de probetas confeccionadas (64), se ensayan a flexotracción un total de 56.28 probetas por cada relación agua cemento $(0,45$ y 0,52$)$.

Docilidad y exudación: en el hormigón fresco se mide la docilidad a través del asentamiento del Cono de Abrams según lo indicado en NCh 1019 Of. 74 [15]. Los valores medios de cada lote se indican en la tabla 3 y se grafican en la figura 6.

Puesto que la trabajabilidad se ve influenciada por la forma y la textura de las partículas que conforman los materiales, las EFC al ser molidas adquieren una forma bastante similar a la de las arenas utilizadas, por lo tanto este factor no afecta a la trabajabilidad de la mezcla. Sin embargo, la textura de las EFC puede considerarse más lisa que la de las arenas, dando como resultado un incremento en la docilidad de la mezcla con respecto al hormigón de referencia, tal como se aprecia en la figura 6.

Una vez colocado el hormigón en las respectivas probetas y luego de realizada la respectiva vibración se observa la presencia de exudación proporcional al contenido de EFC en la mezcla, siendo este efecto mayor en los hormigones con una relación agua cemento de 0,52 [9]. Esto se explica debido al alto peso específico de EFC en relación al resto de los materiales tal como se indica en la tabla $1\left(\mathrm{EFC}=4,0 \mathrm{~kg} / \mathrm{m}^{3}\right.$, Áridos $\left.\cong 2,6 \mathrm{~kg} / \mathrm{m}^{3}\right)$. Por consiguiente, se produce una mayor decantación de esta escoria con respecto al resto de los materiales, provocando un rápido ascenso del agua libre hacia la capa superficial. Adicionalmente se debe tener presente que la absorción de las partículas de $\operatorname{EFC~}(0,2 \%$, tabla 1$)$ es muy baja, lo cual acentúa aún más el fenómeno [7, 9].

En relación a la segregación, no se registró evidencia de este fenómeno mientras se confeccionaron las probetas de hormigón.

Densidad: en el hormigón endurecido se mide la densidad de acuerdo a NCh 1037 Of. 77 [16]. Los valores obtenidos se presentan en la tabla 4 y se grafican en la figura 7.

Se observa que la sustitución de arena por EFC genera un incremento en la densidad, alcanzando valores superiores a $2600 \mathrm{~kg} / \mathrm{m}^{3}$ cuando se utiliza un $50 \%$ de EFC. Lo anterior se atribuye al alto peso específico que presentan las EFC lo que genera un aumento de la densidad media del hormigón. Lo anterior verifica lo reportado por otros investigadores $[4,7]$ en relación a que el peso específico se ve incrementado en el orden de un $10 \%$ con respecto al hormigón de referencia cuando se utiliza un $50 \%$ de EFC.

Tabla 3. Valores medios de la docilidad de acuerdo a NCh 1019 Of. 74 [15].

\begin{tabular}{|c|c|}
\hline Identificación (núm. mediciones) & Docilidad (cm.) \\
\hline$H-0,45-0(4)$ & 3,00 \\
\hline$H-0,45-25(8)$ & 4,75 \\
\hline$H-0,45-40(8)$ & 4,25 \\
\hline$H-0,45-50(8)$ & 4,50 \\
\hline$H-0,52-0(4)$ & 3,50 \\
\hline$H-0,52-25(8)$ & 5,00 \\
\hline$H-0,52-40(8)$ & 4,25 \\
\hline$H-0,52-50(8)$ & 4,45 \\
\hline
\end{tabular}

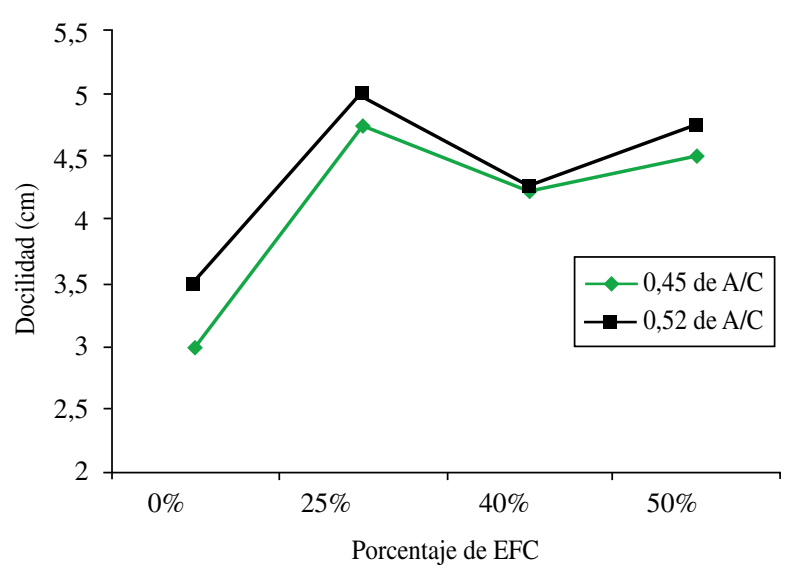

Figura 6. Variación de la docilidad en función del contenido de EFC.

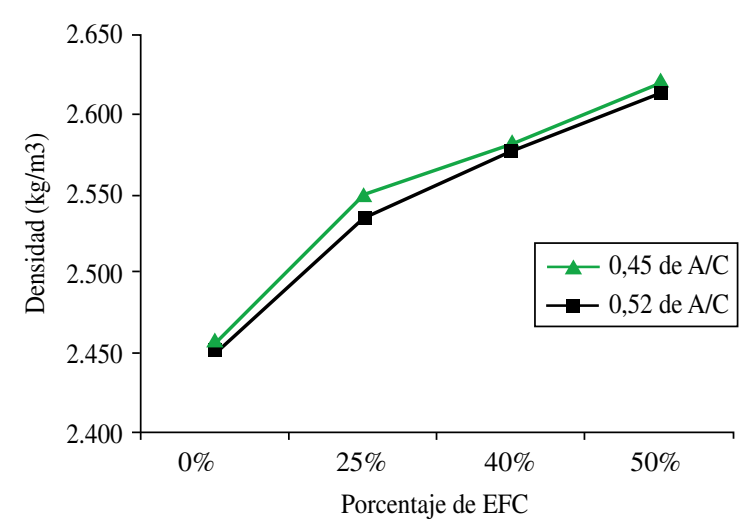

Figura 7. Variación de la densidad media en el estado endurecido en función del contenido de EFC. 
Tabla 4. Densidad, densidad media y desviación normal en $\mathrm{kg} / \mathrm{m}^{3}$ en el hormigón endurecido.

\begin{tabular}{|c|c|c|c|c|}
\hline EFC & $\mathbf{A} / \mathbf{C}$ & $\rho$ & $\rho_{m}$ & $\mathbf{s}$ \\
\hline & \multirow[b]{4}{*}{0,45} & 2.461 & \multirow[b]{4}{*}{2.456} & \multirow[b]{4}{*}{4,57} \\
\hline & & 2.459 & & \\
\hline & & 2.451 & & \\
\hline & & 2.454 & & \\
\hline & \multirow[b]{4}{*}{0,52} & 2.449 & \multirow[b]{4}{*}{2.451} & \multirow[b]{4}{*}{2,36} \\
\hline & & 2.451 & & \\
\hline & & 2.454 & & \\
\hline & & 2.449 & & \\
\hline \multirow{15}{*}{$0 \%$} & \multirow[b]{8}{*}{0,45} & 2.550 & \multirow[b]{8}{*}{2.549} & \multirow[b]{8}{*}{3,62} \\
\hline & & 2.547 & & \\
\hline & & 2.555 & & \\
\hline & & 2.551 & & \\
\hline & & 2.543 & & \\
\hline & & 2.547 & & \\
\hline & & 2.551 & & \\
\hline & & 2.551 & & \\
\hline & \multirow[b]{8}{*}{0,52} & 2.530 & \multirow[b]{8}{*}{2.535} & \multirow[b]{8}{*}{6,78} \\
\hline & & 2.541 & & \\
\hline & & 2.527 & & \\
\hline & & 2.545 & & \\
\hline & & 2.533 & & \\
\hline & & 2.527 & & \\
\hline & & 2.537 & & \\
\hline $25 \%$ & & 2.540 & & \\
\hline \multirow[b]{16}{*}{$40 \%$} & \multirow[b]{8}{*}{0,45} & 2.576 & \multirow[b]{8}{*}{2.582} & \\
\hline & & 2.582 & & \\
\hline & & 2.589 & & \\
\hline & & 2.583 & & \\
\hline & & 2.580 & & \\
\hline & & 2.576 & & \\
\hline & & 2.582 & & \\
\hline & & 2.588 & & 4,81 \\
\hline & & 2.583 & & \\
\hline & & 2.571 & & \\
\hline & & 2.580 & & \\
\hline & & 2.570 & & \\
\hline & & 2.579 & & \\
\hline & & 2.583 & & \\
\hline & & 2.576 & & \\
\hline & 0,52 & 2.570 & 2.577 & 5,58 \\
\hline & & 2.609 & & \\
\hline & & 2.639 & & \\
\hline & & 2.635 & & \\
\hline & & 2.603 & & \\
\hline & & 2.611 & & \\
\hline & & 2.603 & & \\
\hline & & 2.630 & & \\
\hline & 0,45 & 2.636 & 2.621 & 15,7 \\
\hline & & 2.617 & & \\
\hline & & 2.616 & & \\
\hline & & 2.613 & & \\
\hline & & 2.613 & & \\
\hline & & 2.617 & & \\
\hline & & 2.611 & & \\
\hline & & 2.603 & & \\
\hline $50 \%$ & 0,52 & 20 & 2.614 & 5,2 \\
\hline
\end{tabular}

Tabla 5. Carga máxima aplicada $\mathrm{P}(\mathrm{N})$, tensión de rotura $R_{i}$, tensión de rotura media $R_{m}$ en (MPa) de acuerdo con NCh 1038 Of. 77[14] para una relación agua cemento de 0,45 .

\begin{tabular}{|c|c|c|c|c|c|c|c|}
\hline EFC & Edad & $\mathbf{h}$ & b & $\mathbf{P}$ & $\mathbf{R}$ & $\mathbf{R m}$ & $\mathbf{s}$ \\
\hline \multirow[b]{4}{*}{$0 \%$} & 3 & 151 & 151 & 19.260 & 2,52 & 2,52 & \\
\hline & 7 & 151 & 152 & 26.240 & 3,41 & 3,41 & \\
\hline & 28 & 152 & 152 & 38.540 & 4,94 & \multirow[b]{2}{*}{4,96} & \\
\hline & 28 & 153 & 151 & 39.140 & 4,98 & & \\
\hline \multirow[b]{8}{*}{$25 \%$} & 3 & 151 & 152 & 21.020 & 2,73 & \multirow[b]{2}{*}{2,65} & \\
\hline & 3 & 151 & 152 & 19.790 & 2,57 & & \\
\hline & 7 & 151 & 151 & 26.840 & 3,51 & \multirow[b]{2}{*}{3,48} & \\
\hline & 7 & 152 & 152 & 26.870 & 3,44 & & \\
\hline & 28 & 151 & 153 & 39.230 & 5,06 & \multirow{4}{*}{\multicolumn{2}{|c|}{5,10}} \\
\hline & 28 & 152 & 151 & 39.960 & 5,15 & & \\
\hline & 28 & 151 & 153 & 39.580 & 5,11 & & \\
\hline & 28 & 152 & 153 & 39.670 & 5,05 & & \\
\hline \multirow[b]{8}{*}{$40 \%$} & 3 & 151 & 152 & 22.360 & 2,90 & \multirow[b]{2}{*}{2,80} & \\
\hline & 3 & 152 & 153 & 21.040 & 2,68 & & \\
\hline & 7 & 152 & 152 & 29.540 & 3,79 & \multirow[b]{2}{*}{3,81} & \\
\hline & 7 & 152 & 152 & 29.820 & 3,82 & & \\
\hline & 28 & 152 & 152 & 41.920 & 5,37 & \multirow{4}{*}{\multicolumn{2}{|c|}{0,03}} \\
\hline & 28 & 151 & 152 & 41.220 & 5,35 & & \\
\hline & 28 & 153 & 152 & 42.350 & 5,36 & & \\
\hline & 28 & 152 & 151 & 41.970 & 5,41 & & \\
\hline \multirow[b]{8}{*}{$50 \%$} & 3 & 151 & 152 & 20.100 & 2,61 & \multirow[b]{2}{*}{2,64} & \\
\hline & 3 & 151 & 150 & 20.290 & 2,67 & & \\
\hline & 7 & 151 & 151 & 26.700 & 3,49 & \multirow[b]{2}{*}{3,57} & \\
\hline & 7 & 152 & 153 & 28.690 & 3,65 & & \\
\hline & 28 & 153 & 152 & 41.840 & 5,29 & \multirow[b]{4}{*}{5,28} & \multirow[b]{4}{*}{0,02} \\
\hline & 28 & 151 & 152 & 40.610 & 5,27 & & \\
\hline & 28 & 151 & 150 & 40.370 & 5,31 & & \\
\hline & 28 & 151 & 150 & 40.010 & 5,26 & & \\
\hline
\end{tabular}

Carga y tensión de rotura en flexotracción: La evolución de la resistencia media a la flexotracción $R_{m}$ en (MPa) para cada edad de carga ( 3,7 y 28 días) a igual contenido de $\operatorname{EFC}(0 \%, 25 \%, 40 \%$ y $50 \%)$ para cada relación agua cemento $(0,45$ y 0,52$)$, se presenta en la tabla 5 y se grafica en las figuras 8 y 9 .

Análisis de resultados: De tablas 5 y 6 y de figuras 8 y 9 , se concluye que se presenta un aumento en la resistencia media a flexotracción a medida que se incrementa el porcentaje de EFC utilizado como árido fino. 
Tabla 6. Carga máxima aplicada $\mathrm{P}(\mathrm{N})$, tensión de rotura $R_{i}$, tensión de rotura media $R_{m}$ en $(\mathrm{MPa})$ de acuerdo con NCh 1038 Of. 77 [14] para una relación agua cemento de 0,52 .

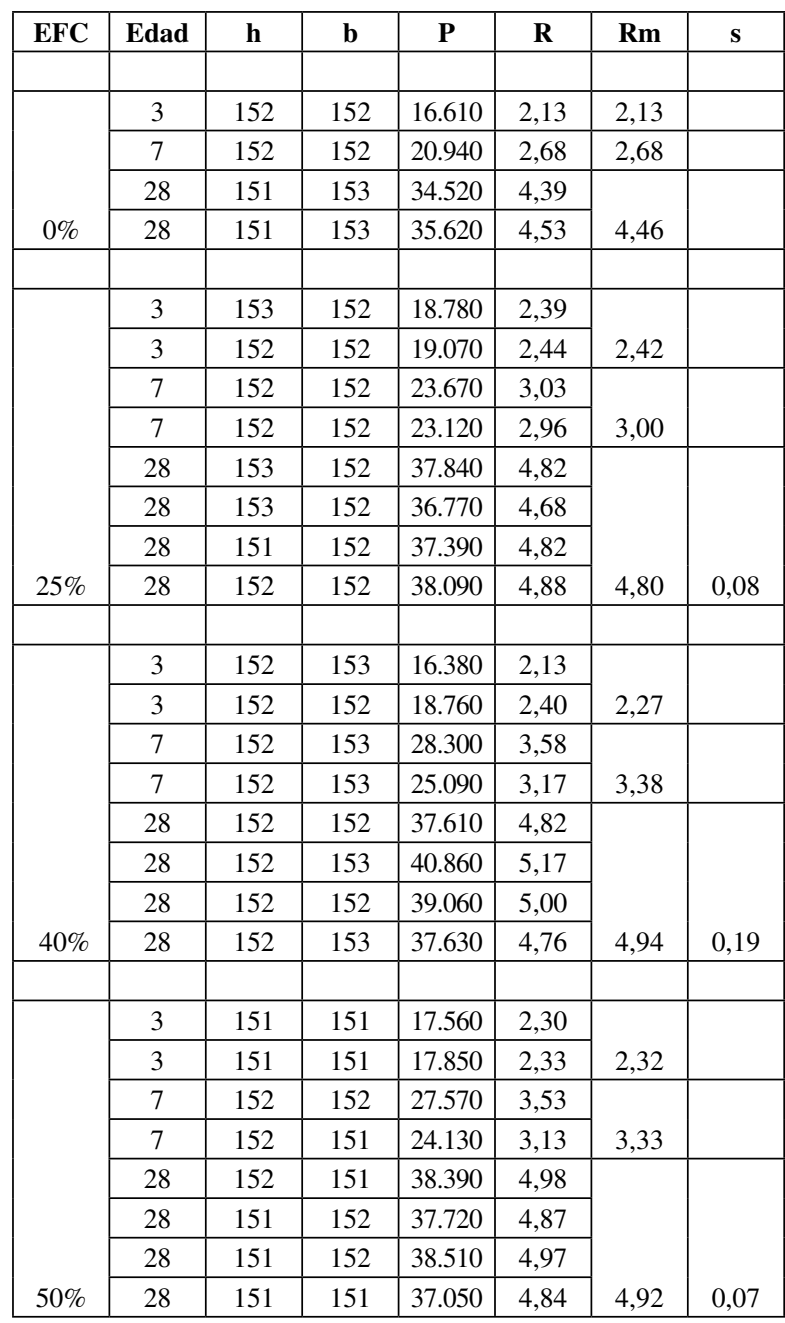

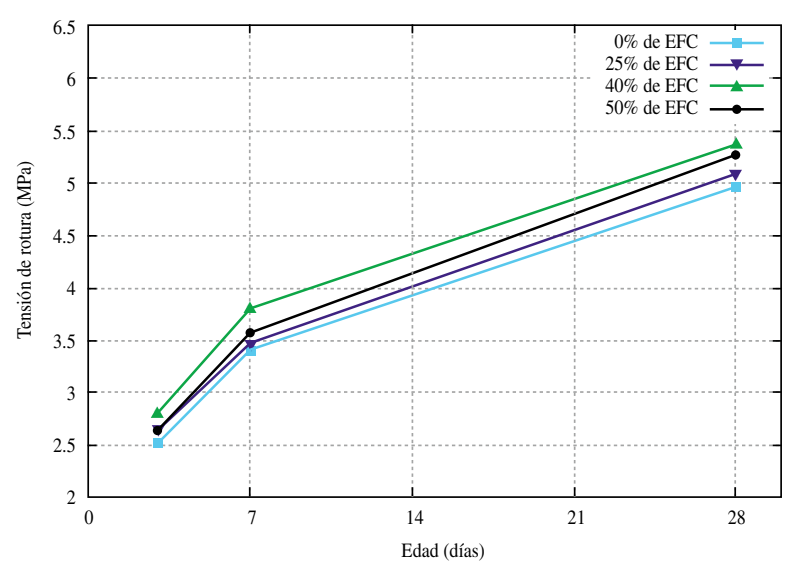

Figura 8. Evolución de la resistencia media a flexotracción en función del contenido de EFC para una relación agua cemento de 0,45 .

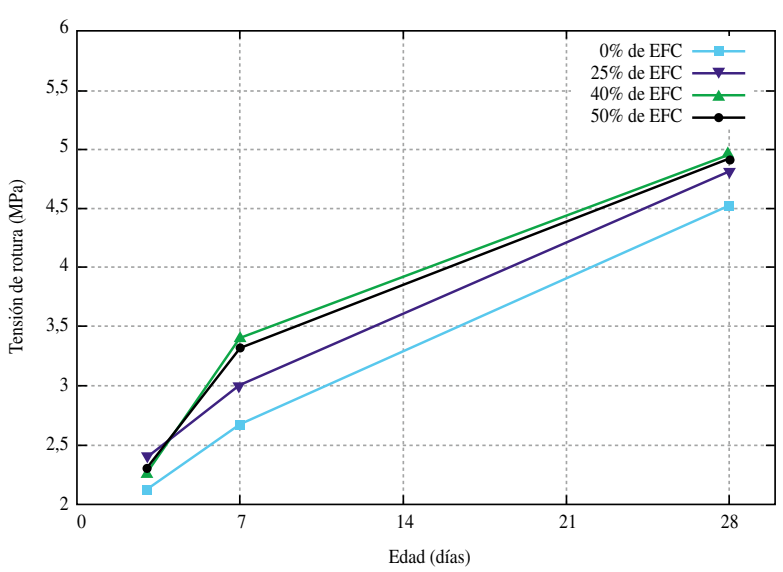

Figura 9. Evolución de la resistencia media a flexotracción en función del contenido de EFC para una relación agua cemento de 0,52 .

Los valores máximos de la resistencia se alcanzan para un contenido de EFC del 40\%, sin embargo, dichos valores son muy cercanos a los obtenidos con un $50 \%$ de tal como lo indica el análisis de la desviación normal de dichas medias. No obstante, queda de manifiesto que se produce un aumento de la resistencia a la flexotracción con respecto al hormigón de referencia.

En el caso de utilizar una relación agua cemento de 0,45 se genera un incremento del orden del 8,3\% cuando se utiliza un $40 \%$ de EFC y de un 6,5\% con un 50\% de EFC. Lo mismo ocurre para una relación agua cemento de 0,52 , en este caso se logra un incremento del $10,8 \%$ para un $40 \%$ de EFC y de un $10,3 \%$ para un $50 \%$ de contenido de EFC. Moura [4], en su investigación, concluye que la resistencia a la flexotracción se ve incrementada en un orden del $10 \%$ con respecto al hormigón de referencia cuando se utiliza un porcentaje de reemplazo de $40 \%$ de escoria.

Carga y tensión de rotura en compresión: de forma de estudiar lo que sucede con la resistencia a la compresión, los trozos de viguetas obtenidos del ensayo de flexotracción se preparan y someten al ensayo de compresión de acuerdo con NCh 1037 Of. 77 [16]. Dado que los trozos de viguetas no tienen dimensiones normalizadas, es necesario colocarlas dentro de un molde (ver figura 10), de modo que la carga de compresión se aplique a través de placas rígidas ubicadas en la cara superior e inferior de dicho molde. 


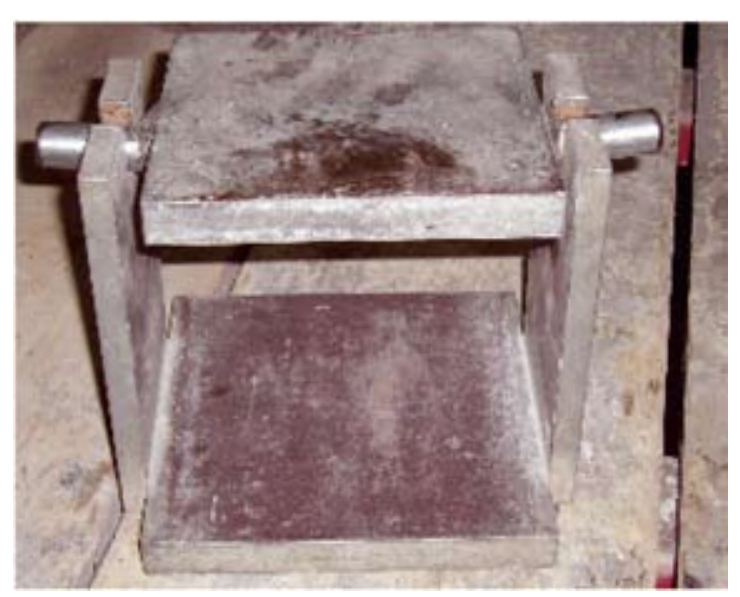

Figura 10. Esquema de molde utilizado para realizar ensayo a compresión de trozos de viguetas.

En las tablas 7 y 8 se presentan para cada una de las probetas ensayadas el valor de la carga máxima de compresión axial aplicada $P_{i}(\mathrm{~N})$, la tensión de rotura por compresión $R_{i}(\mathrm{MPa})$ y su valor medio calculado según ecuación (2).

$$
R_{i}=\frac{P_{i}}{S}
$$

En donde:

$S=$ Superficie de carga $(155 \mathrm{~mm} \times 152 \mathrm{~mm})$.

La evolución de la resistencia media a la compresión en función de la relación agua cemento y el contenido de $\mathrm{EFC}$, se presenta en las figuras 10 y 11.

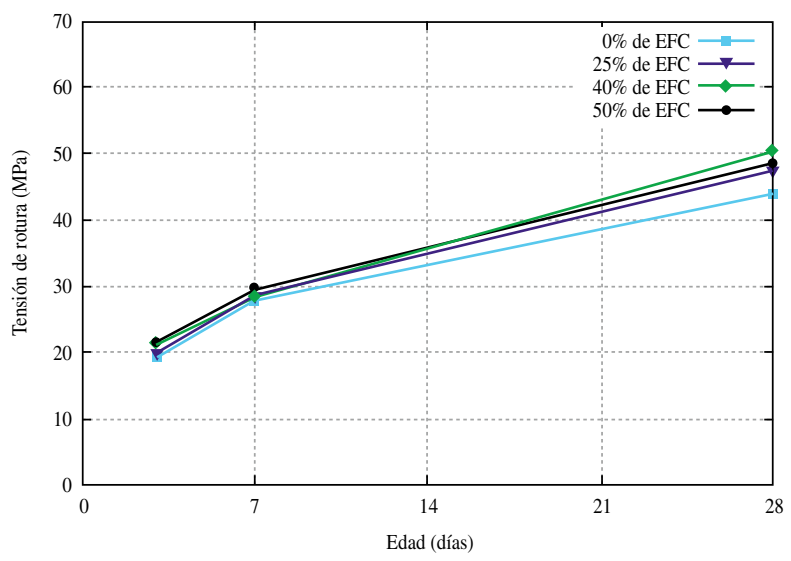

Figura 10. Evolución de la tensión de rotura en compresión en función del contenido de EFC para una relación agua cemento de 0,45 .

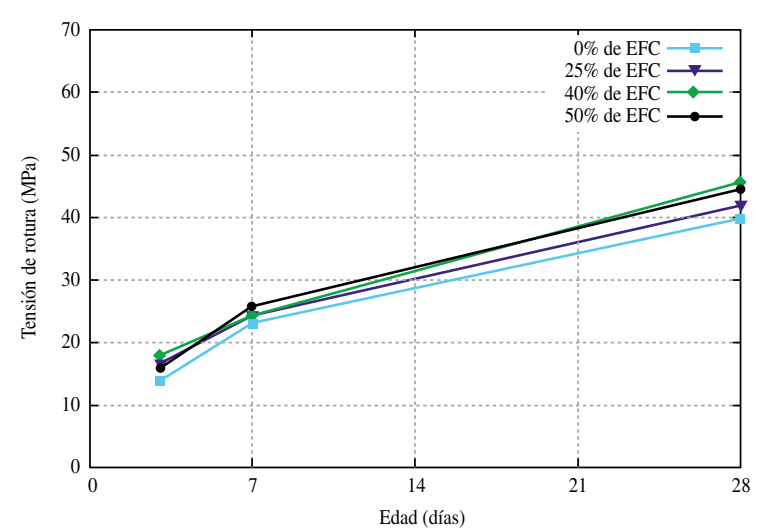

Figura 11. Evolución de la tensión de rotura en compresión en función del contenido de EFC para una relación agua cemento de 0,52.

Tabla 7. Carga máxima aplicada $\mathrm{P}(\mathrm{N})$, tensión de rotura a compresión, tensión media de rotura a compresión y desviación normal en (MPa) de acuerdo con NCh 1037 Of. 77 [16] para una relación agua cemento de 0,45 .

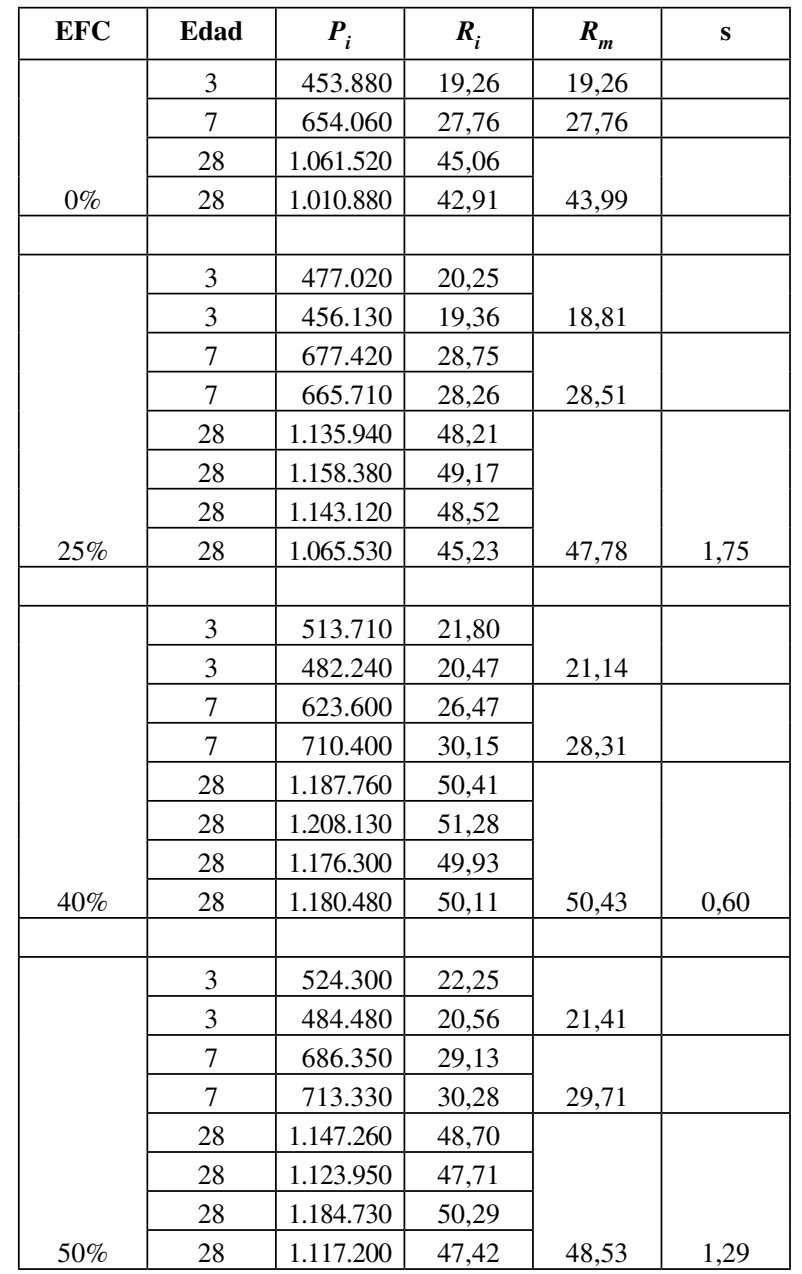


Tabla 8. Carga máxima aplicada $\mathrm{P}(\mathrm{N})$, tensión de rotura a compresión, tensión media de rotura a compresión y desviación normal en (MPa) de acuerdo con NCh 1037 Of. 77[16] para una relación agua cemento de 0,52.

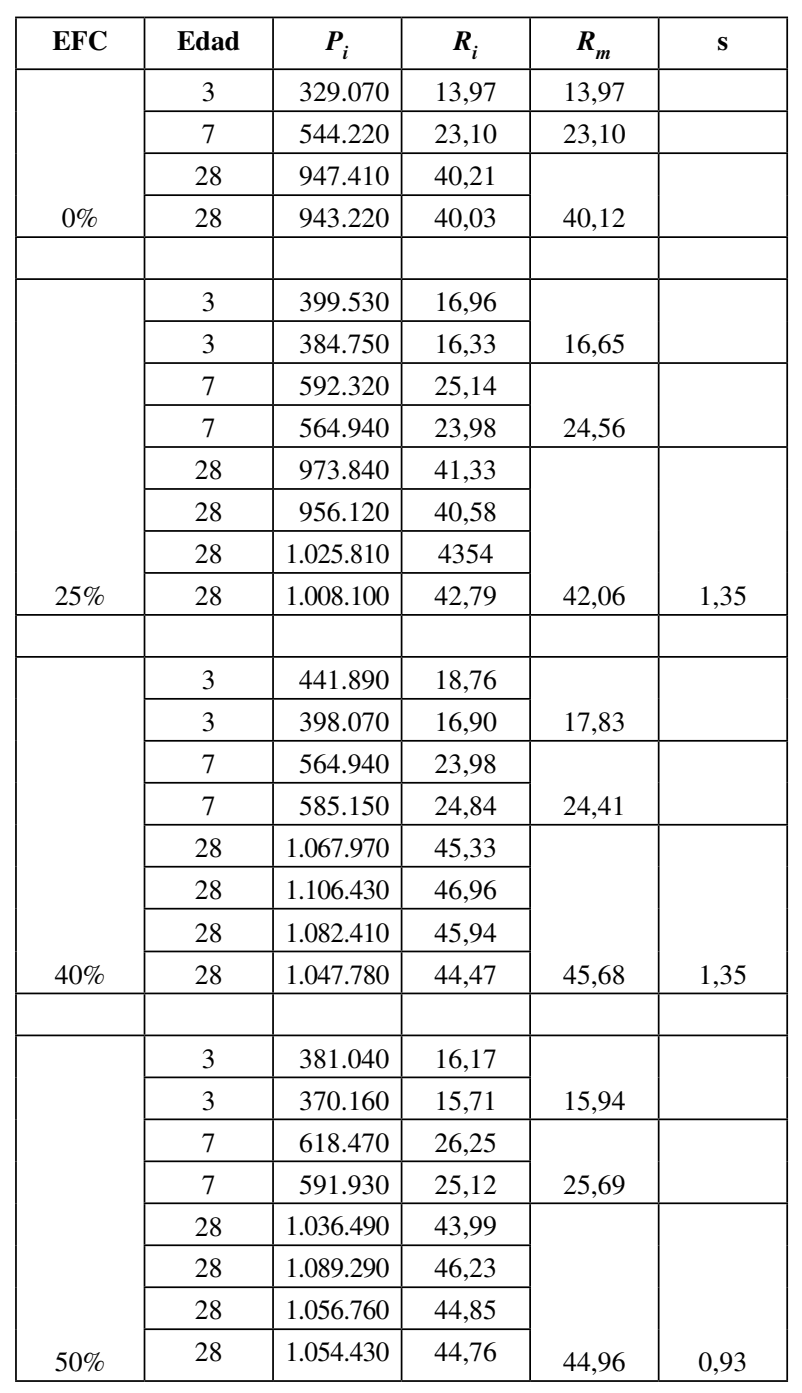

De las figuras 10 y 11 se concluye que el desarrollo de resistencia a compresión a edades tempranas (3, 7 días) es muy similar para todos los contenidos de EFC y una relación agua cemento; sin embargo a edades mayores se produce un aumento de la resistencia en relación al hormigón de referencia, llegando a valores del 14,6\% en el caso de considerar un $40 \%$ de EFC y una relación agua cemento de 0,45 . Al igual que en caso de flexotracción y considerando los valores de las desviaciones normales es difícil concluir cual de los porcentajes de EFC (40\% o 50\%) genera la máxima tensión de rotura a la compresión.
Los resultados obtenidos concuerdan con lo expuesto por investigaciones anteriores [4, 7-8] en relación a que la resistencia a la compresión axial se ve incrementada con la incorporación de EFC, independientemente de la edad de las probetas y razones agua cemento ensayadas.

\section{CONCLUSIONES}

Dentro de las principales conclusiones obtenidas en el marco de la presente investigación, se destacan:

1. La incorporación de EFC afecta la trabajabilidad de la mezcla, se observa un incremento de la docilidad del hormigón con contenido de EFC en relación al hormigón de referencia. Esto se atribuye a la textura de las EFC que resultan ser más lisas que la de las arenas utilizadas.

2. Se observa un aumento de la exudación en los hormigones que contienen EFC con respecto al hormigón de referencia, siendo este proporcional al contenido de las mismas. Este incremento se atribuye al alto peso específico de EFC en relación al resto de los materiales y a que la absorción de las partículas de EFC es muy baja.

3. En el hormigón endurecido se observa que sustitución de arena por un determinado porcentaje de EFC genera un incremento proporcional en la densidad del hormigón, alcanzando valores superiores a 2600 $\mathrm{kg} / \mathrm{m}^{3}$ cuando se utiliza un $50 \%$ de EFC. Lo anterior se atribuye al alto peso específico que presenta la escoria, la cual genera un aumento de la densidad media a medida que se incrementa el porcentaje de EFC.

4. La resistencia a la flexotracción y compresión del hormigón aumenta en todos los casos estudiados, en función del porcentaje de incorporación de EFC. Se concluye que la principal ventaja de las EFC desde el punto de vista de la resistencia es el incremento de la capacidad de carga con respecto al hormigón de referencia.

5. Los valores máximos de la resistencia tanto a flexotracción como compresión se alcanzan para contenidos de EFC del $40 \%$ y $50 \%$. Sin embargo, después de un análisis de la desviación normal de los valores medios no es posible concluir cual de ambos contenidos de EFC genera la tensión de rotura mayor. 


\section{REFERENCIAS}

[1] O. Pavez, J. Palacios and M. Sánchez. "A review of copper pyrometallurgical slags utilization". 5th International Symposium on Waste processing and recycling in the mineral and metallurgical industries. Hamilton, Ontario, Canada, pp. 291-298. August 22 to $25,2004$.

[2] M. Sánchez, F. Parada, R. Parra, F. Márquez, R. Jara, J.C. Carrasco and J. Palacios. "Management of copper pyrometallurgical slags: giving additional value to the copper mining industry". VII Int. Conference on Molten Slags, Fluxes \& Salts, Cape Town, South Africa, pp. 543-550. 25-28 January, 2004.

[3] A. Ariño and B. Mobasher. "Effect of copper slag on the strength, and toughness of cementitious mixtures". ACI Materials Journal, pp. 68-75. January-February, 1999.

[4] W. Moura and D. Coutinho. "Influence of copper slag admixture in concrete in durability properties". Ambiente Construido. Porto Alegre. Vol. $4 \mathrm{~N}^{\mathrm{o}} 2$, pp. 41-56. April 2004.

[5] JIS Technical Report, TRA 0006. "Recycled Concrete Using Recycled Aggregate”. Public Works Research Institute. Japan. 2000.

[6] K. Al-Jabri, R. Taha and M. Al- Ghassani. "Use of copper slag and cement by-pass dust as cementitious materials". Cement Concrete Aggr Vol. $24 \mathrm{~N}^{\mathrm{o}}$ 1, pp. 7-12. 2002.

[7] M. Zapata. "Caracterización de cementos y hormigones fabricados a partir de escorias de fundición de cobre". Tesis para optar al título de Ingeniero Civil. Departamento de Ingeniería Civil. Facultad de Ingeniería. Universidad de Concepción, p. 173. 2006.
[8] P. Cendoya y M. Liberona. "Efecto de las escorias de fundición de cobre como agregado fino en las propiedades del hormigón fresco y endurecido". XVI Jornadas Chilenas del Hormigón. Universidad Católica del Maule, Talca. 17-19 octubre, 2007.

[9] L. Cares. "Análisis teórico-experimental de la resistencia a la flexotracción de hormigones con escoria de fundición de cobre". Tesis para optar al Título de Ingeniero Civil. Departamento de Ingeniería Civil. Facultad de Ingeniería. Universidad de Concepción, p. 167. 2007.

[10] NCh 163 Of. 79. “Áridos para morteros y hormigones-Requisitos generales". Instituto Nacional de Normalización. Chile.

[11] NCh 148 Of. 68. "Cemento, terminología, clasificación y especificaciones generales”. Instituto Nacional de Normalización. Chile.

[12] NCh 170 Of. 85. "Hormigón-Requisitos generales". Instituto Nacional de Normalización. Chile.

[13] NCh 1017 Of. 75. "Hormigón-Confección y curado en obra de probetas para ensayos de compresión y tracción”. Instituto Nacional de Normalización. Chile.

[14] NCh 1038 Of. 77. "Hormigón-Ensayo de tracción por flexión". Instituto Nacional de Normalización. Chile.

[15] NCh 1019 Of. 74. "Construcción-HormigónDeterminación de la docilidad-Método del asentamiento del cono de Abrams". Instituto Nacional de Normalización. Chile.

[16] NCh 1037 Of. 77. "Hormigón-Ensayo de compresión de probetas cúbicas y cilíndricas”. Instituto Nacional de Normalización. Chile. 